\title{
The locality of the fourth root of staggered fermion determinant in the interacting case.
}

\section{Bernard}

Department of Physics, Washington University, St. Louis, MO 63130, USA

E-mail: cb@lump.wustl.edu

C. DeTar and F. Maresca*

Department of Physics, University of Utah, Salt Lake City, UT 84112, USA

E-mail: detar@physics.utah.edu, maresca@physics.utah.edu

\section{Steven Gottlieb and L. Levkova}

Department of Physics, Indiana University, Bloomington, IN 47405, USA

E-mail: sg@physics.indiana.edu, l levkova@indiana.edu

\section{U.M. Heller}

American Physical Society, One Research Road, Box 9000, Ridge, NY 11961, USA

E-mail: hellereaps.org

\section{J.E. Hetrick}

Physics Department, University of the Pacific, Stockton, CA 95211, USA

E-mail: jhetrick@pacific.edu

\section{R. Sugar}

Department of Physics, University of California, Santa Barbara, CA 93106, USA

E-mail: sugar@vulcan2. physics .ucsb.edu

\section{Renner and D. Toussaint}

Department of Physics, University of Arizona, Tucson, AZ 85721, USA

E-mail: doug@physics.arizona.edu, dru@physics.arizona.edu

The fourth root approximation in LQCD simulations with dynamical staggered fermions requires justification. We test its validity numerically in the interacting theory in a renormalization group framework.

XXIIIrd International Symposium on Lattice Field Theory

25-30 July 2005

Trinity College Dublin, Ireland

\footnotetext{
${ }^{*}$ Speaker.
} 


\section{Introduction}

Numerical simulations of dynamical fermions within the framework of the staggered formalism are both computationally cost effective and phenomenologically successful. Exploiting the advantageous properties of an improved staggered fermion formulation, various collaborations are performing high-precision lattice QCD calculations that are in excellent agreement with experimentally known measurements [1]. However, this success is clouded by the long-standing problematic issue of the validity of the fourth root approximation. The staggered fermion describes, in fact, four tastes on the lattice, so in order to study QCD with $N_{f}=2$ or $N_{f}=2+1$, a Boltzmann weighting is used that contains a fractional power of the fermion determinant. Because of tastebreaking at nonzero lattice spacing, taking the fractional power of the staggered determinant before restoring taste symmetry is conceptually nontrivial. The issue whether the fourth root prescription gives a lattice theory in the right universality class to reproduce QCD is yet unresolved and the phenomenological success of the staggered formulation has heated up the discussion. In the past years various numerical investigations [2] have addressed this concern. Recently a direct approach was adopted towards establishing whether the universality class is the right one: if a single-taste local fermion action can be found, whose determinant is equal to the fourth root of the staggered fermion determinant up to cutoff effects

$$
\lim _{a \rightarrow 0}\left(\operatorname{det} D_{s t}\right)^{1 / 4}=\operatorname{det} D \operatorname{det} H
$$

(where $D$ is a local Dirac operator and $H$ is local and contains only cutoff effects) then the fourth root prescription can be consistently fitted into the framework of a local field theory. In the free theory numerical [3] and analytical studies [4, 5] showed that such a local operator exists. In particular, in [5] Shamir applied renormalization group blocking to the free staggered operator $D_{0}$ in the spin $\otimes$ taste representation. After $n$ blocking steps $Q_{n}$, the fermionic degrees of freedom live on the coarse lattice with lattice spacing $a_{c}=2^{n} a$ and the determinant of the staggered operator decomposes as det $D_{s t}\left(a=2^{-n} a_{c}\right)=\operatorname{det} D_{n} \operatorname{det} G_{n}^{-1}$. More details can be found in [5], where it was proved analytically that the blocked propagator $D_{n}^{-1}=\alpha^{-1}+Q_{n} D_{0}^{-1} Q_{n}^{\dagger}$ factorizes, in the limit $n \rightarrow \infty$, as $D_{\infty}^{-1}=D_{\operatorname{rg}} \otimes I$ and that $G_{n}^{-1}=D_{0}+\alpha Q_{n}^{\dagger} Q_{n}$ is a local operator that contains only cutoff effects. This completes the proof that in the continuum limit, i.e. $a\left(=2^{-n} a_{c}\right) \rightarrow 0$, the decompositions of Eq. (1.1) holds in the free case.

\section{Renormalization group transformation in the interacting case}

In this section we briefly discuss the renormalization group (RG) program adopted to test the interacting case. The new complication is that a mapping from the one-component staggered fermion basis to the spin $\otimes$ taste basis is not unique. We define the interacting theory in the one-component formalism. The first fermion RG blocking transformation is used to perform the transition to the spin $\otimes$ taste representation:

$$
\left(Q_{(1)} \psi\right)^{a \alpha}(2 x)=\frac{1}{2} \sum_{r_{\mu}=0,1}\left(\gamma_{1}^{r_{1}} \gamma_{2}^{r_{2}} \gamma_{3}^{r_{3}} \gamma_{4}^{r_{4}}\right)^{(a \alpha)} W_{r}(2 x, 2 x+r) \psi(2 x+r),
$$


where $a$ and $\alpha$ are Dirac spin and taste indices respectively. We require the parallel transporters $W_{r}(2 x, 2 x+r)$ to be a sum over all the shortest paths ${ }^{1}$ between the origin $2 x$ and the other sixteen sites $2 x+r$ of the hypercube. The sum over different paths is thought to reduce the breaking of hypercubic symmetry; however, the choice to parallel transport all the points within a given hypercube to the corner $r=0$ unavoidably introduces some asymmetries. The subsequent $\mathrm{RG}$ steps are a covariant generalization of the arithmetic mean on a $2^{4}$ hypercube used in [5] :

$$
\left(Q_{(n)} \psi\right)(2 x)=2^{-4} \sum_{r_{\mu}=0,1} W_{r}(2 x, 2 x+r) \psi(2 x+r) \quad n>1 .
$$

The shortest paths that compose the parallel transporters $W_{r}(2 x, 2 x+r)$ in Eq. (2.2) are constructed from links of the blocked lattice (with lattice spacing $2^{n} a$ ). These blocked links are built following a program suggested in [7]. In detail, the links that live on the fine lattice (with lattice spacing $\left.2^{n-1} a\right)$ are twice APE smeared and projected back to $S U(3)$. The blocked links are then built multiplying two of these fine smeared links in line. Once the blocking kernels are defined we project the nondiagonal piece of the blocked propagator $D_{n}^{-1}$ onto the spin $\otimes$ taste Clifford space:

$$
\left(Q_{n} D_{0}^{-1} Q_{n}^{\dagger}\right)(x, y=0)=\sum_{S, T} \Gamma_{S} \otimes \Gamma_{T}^{\dagger} M_{S T}(x, y=0) .
$$

Here $x$ and $y=0^{2}$ live on the coarsest lattice and $Q_{n}$ can be regarded as a 'big' blocking step $Q_{n}=Q_{(n)} Q_{(n-1)} \cdots Q_{(1)}$ that transforms the original lattice with lattice spacing $a$ into the blocked lattice with lattice spacing $a_{c}=2^{n} a$. The coefficients $M_{S T}(x, y=0)$ of the projection in Eq. (2.3) are evaluated numerically and averaged over two different lattice ensembles. We fixed Lorentz gauge before doing any RG blocking. Parameters of the simulations are shown in Table 1. Applying a different number of RG steps, $n$, on each of these two ensembles, the resulting blocked lattices have the same coarse-lattice spacing of $a_{c}=0.72 \mathrm{fm}$. This allowed us to see how the coefficients $M_{S T}$ scale with one additional RG blocking step.

\begin{tabular}{|l|l|l|}
\hline unblocked lattice & $16^{3} \times 48$ & $40^{3} \times 96$ \\
\hline$a$ & $0.18 \mathrm{fm}$ & $0.09 \mathrm{fm}$ \\
\hline$m_{s} a$ & 0.125 & 0.05 \\
\hline number of RGT's & 2 & 3 \\
\hline blocked lattice & $4^{3} \times 12$ & $5^{3} \times 12$ \\
\hline$a_{c}$ & $0.72 \mathrm{fm}$ & $0.72 \mathrm{fm}$ \\
\hline number of cfgs & 148 & 56 \\
\hline
\end{tabular}

Table 1: Simulation parameters

As a consistency check on the blocking procedure described above, we evaluated the mass of the $(s \bar{s})$ meson. In fact, the spectrum on the blocked lattice is expected to be identical to the spectrum in the original theory. The meson mass can be evaluated directly from the coefficients $M_{S T}(x, y)$ of Eq. (2.3):

\footnotetext{
${ }^{1}$ These are built using APE smeared links of the original lattice to be consistent with the RG program adopted for the subsequent RG steps.

${ }^{2}$ The fermion source is defined at the origin.
} 


$$
C_{s \bar{s}}^{T}(t)=\sum_{S, T^{\prime}, \vec{x}}<\operatorname{Tr}\left(\tau_{5} \Gamma_{T} \Gamma_{T^{\prime}} \Gamma_{T} \tau_{5} \Gamma_{T^{\prime}}^{\dagger}\right)\left|M_{S T^{\prime}}(t, \vec{x})\right|^{2}>
$$

where the $\Gamma_{S}$ 's and the $\Gamma_{T, T^{\prime}}$ 's are the 16 Dirac matrices in the normal and adjoint $\left(\tau_{\mu}=\gamma_{\mu}^{\star}\right)$ representation respectively. In Figure 1 we present the taste splittings for the meson masses evaluated on the two blocked lattices. At this level of statistics, the decrease in splitting from $n=2$ to $n=3$ is consistent with the expected $\mathscr{O}\left(a^{2} \alpha\right)$ or $\mathscr{O}\left(a^{2} \alpha^{2}\right)$, but the preliminary RG blocked splittings are systematically higher than values obtained in direct measurements at much lighter valence quark masses. Further study is needed.

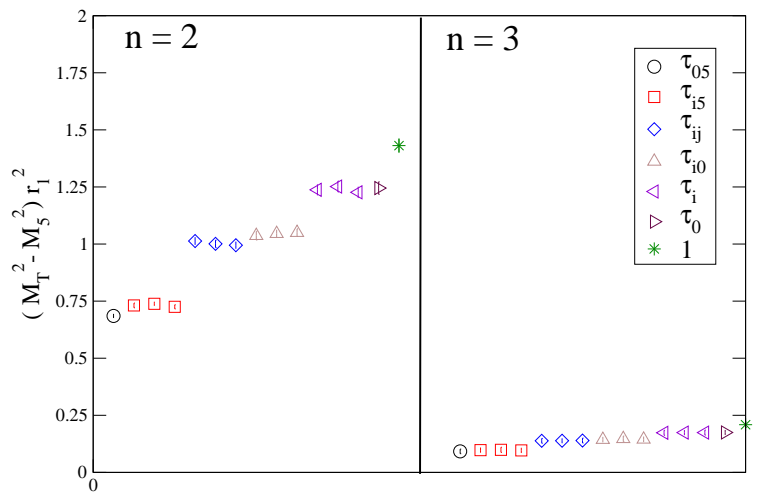

Figure 1: Pseudoscalar taste splittings after 2 and 3 RG blockings in units of $r_{1}$, the distance at which the static quark potential satisfies $r^{2} d V / d r=1$.

\section{Preliminary results}

In this preliminary study we have analyzed for various displacements $|x-y|$ the dominant terms in Eq. (2.3) that are also present in the free theory:

$\sum_{\mu}\left(\gamma_{\mu} \otimes I\right) A_{\mu}^{(n)}(x, y)+\left(\gamma_{5} \otimes \tau_{5} \tau_{\mu}\right) B_{\mu}^{(n)}(x, y)+\sum_{v \neq \mu}\left(\gamma_{5} \otimes \tau_{5} \tau_{v}\right) B_{v}^{(n)}(x, y)+(I \otimes I) C^{(n)}(x, y)+\cdots \cdots$

Comparing Figures 2 and 3, it is remarkable to see that increasing by only one unit the number of RG blocking transformations the mass term $(I \otimes I)(\square)$ becomes larger than the taste breaking term $\gamma_{5} \otimes \tau_{5} \tau_{i}(\diamond)$ for any direction $i$ for displacement $|x-y|=1$. This is in agreement with what we expect from the free theory: in the limit $n \rightarrow \infty$ the only terms in $M_{S T}$ that should survive are diagonal in taste space.

Other terms in Eq. (3.1) are seen in our simulations. Their contribution is $10-1000$ times smaller than the leading kinetic term $A_{\mu}^{(n)}$ in Eq. (3.1) and this make it difficult to identify the terms that are statistically relevant. We need to increase the number of configurations to have a better understanding of the statistics. Besides this issue, it is crucial to know how these terms scale with $n$ to determine whether the blocked propagator becomes diagonal in taste space in the interacting theory as it does in the free theory. However an element that can play an important role in this scaling is the breaking of the hypercubic symmetry, caused by the introduction of the parallel transporters, as it has been briefly discussed in the previous section. In fact, Figures 2 
Displacement of 1

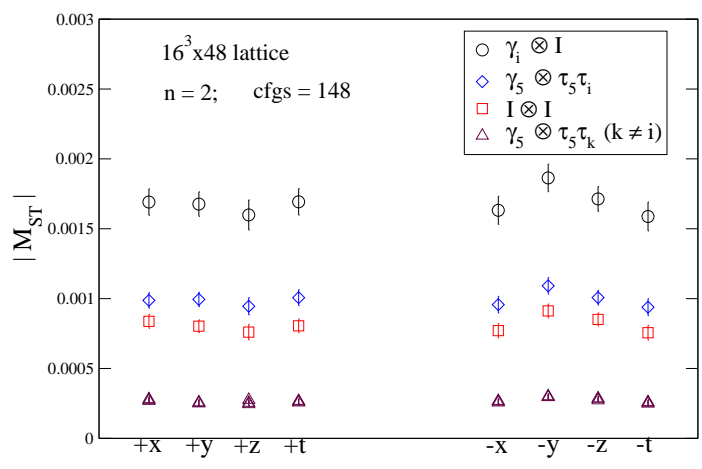

$(+)$ (i)

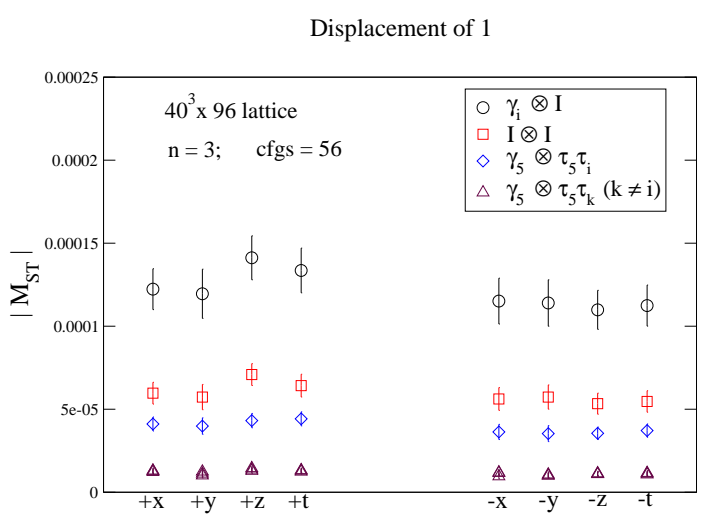

$(+)$

$(-)$

Figure 3: $M_{S T}$ after $n=3 \mathrm{RG}$ steps.

Figure 2: $M_{S T}$ after $n=2 \mathrm{RG}$ steps.

and 3 suggest that the blocking procedure is introducing some hypercubic asymmetries, since the coefficients $M_{S T}(x, y)$ are not perfectly degenerate under reflections about the principal axes. In order to investigate further these terms, present only in the interacting theory, it is necessary either to quantify the hypercubic symmetry breaking or to redefine the RG blocking transformations in order to guarantee hypercubic invariance (one suggestion is presented at this conference [6]).

\section{Interacting theory vs free theory}

In [5] the scaling properties of the taste breaking terms $B_{\mu}^{(n)}(p)$ were evaluated analytically. It was shown in momentum space that they scale like $2^{-n}$, uniformly in $p$, so the blocked propagator becomes diagonal in taste space, when $n \rightarrow \infty$. Postponing the issue of the breaking of hypercubic symmetry to a later study, we consider here a linear combination of the amplitudes $\left|M_{S T}(x, y)\right|$ under reflections and rotations about the principal axes. In Figures 4 and 5 we show how the ratios between the taste violating terms and the leading kinetic term $A^{(n)}$ scale with $n$.

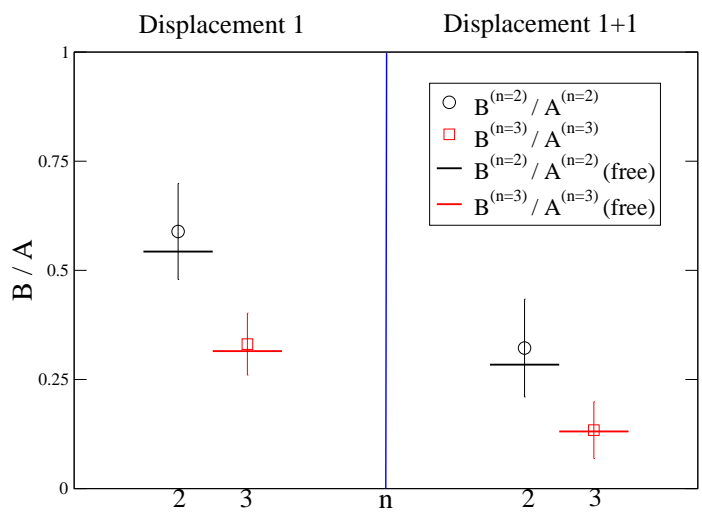

Figure 4: Taste breaking term $B^{(n)}$ over the kinetic term.

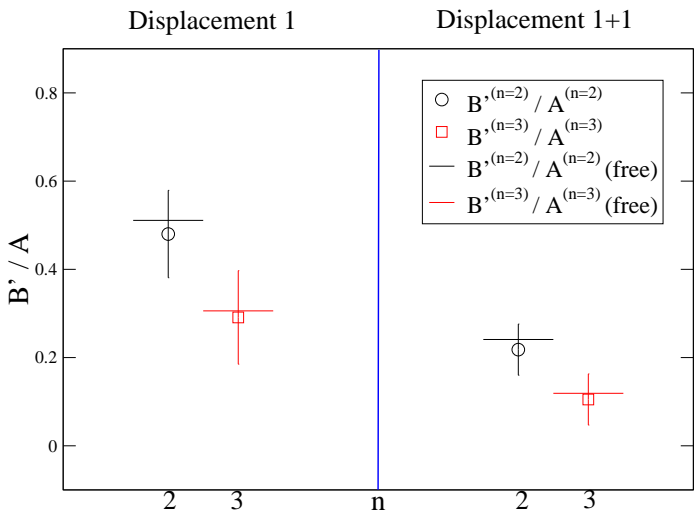

Figure 5: Taste breaking term $B^{\prime(n)}$ over the kinetic term.

Our data show that they diminish when $n$ is increased and that their magnitudes are also remarkably in agreement with the free theory represented by solid lines. In Figures 6 and 7 we show 
the scaling properties of the taste breaking term divided by the mass term. The interacting theory agrees with the free theory for displacements $|x-y|=1,2$, however a discrepancy is seen at zero displacement where the interacting theory does not scale as expected. The zero displacement is not interesting when discussing locality thus this behavior, even if not well understood, does not spoil the good scaling properties we are seeing in the interacting theory.

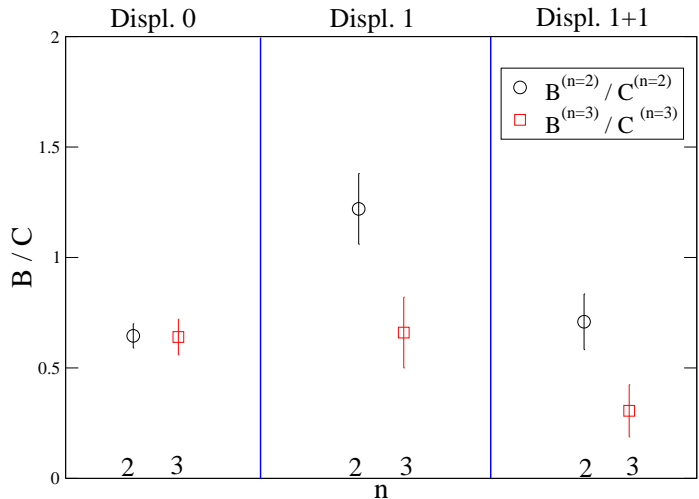

Figure 6: Taste breaking term divided by the mass term: interacting theory

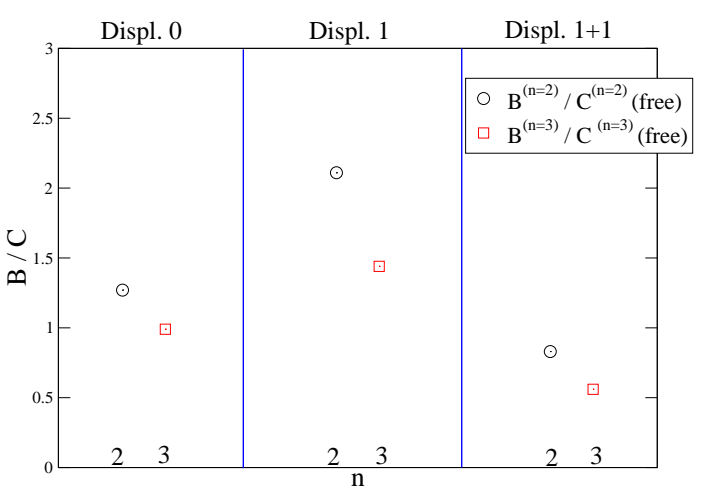

Figure 7: Taste breaking term divided by the mass term: free theory.

The conclusion that we can draw from this preliminary study is that the blocked propagator in the interacting case is dominated by the same terms present in the free theory and that these terms scale as expected to make the decomposition of Eq. (1.1) possible. The statistical relevance and the scaling properties of other terms seen in the interacting theory is still under investigation.

We thank Yigal Shamir and Maarten Goltermann for helpful discussions during the conference. Computing support from the Utah Center for High Performance Computing is gratefully acknowledged. This work was supported by the US NSF and DOE.

\section{References}

[1] C.T.H. Davies et al.[HPQCD, UKQCD and MILC Collaborations] High-precision lattice QCD confronts experiment, Phys.Rev.Lett.92:022001,2004, [hep-lat/0304004]

[2] S. Dürr and C. Hoelbling, Scaling tests with dynamical overlap and rooted staggered fermions, Phys.Rev.D71:054501,2005, [hep-lat/0411022]; E. Follana et al., The index theorem and universality properties of the low-lying eigenvalues of improved staggered quarks, Phys.Rev.Lett.93:241601,2004, [hep-lat/0406010]

[3] F. Maresca and M. Peardon, A path-integral representation of the free one-flavor staggered-fermion determinant, [hep-lat/0411029]

[4] D. Adams, On the fourth root prescription for dynamical staggered fermions, [hep-lat/0411030]

[5] Y. Shamir, Locality of the fourth root of the staggered fermion determinant: renormalization-group approach, Phys.Rev.D71:034509,2005, [hep-lat/0412014]

[6] Y. Shamir, These proceedings.

[7] T. DeGrand et al. The classically perfect fixed point action for $S U(3)$ gauge theories, Nucl.Phys.B454:587-614,1995, [hep-lat/9506030] 